\title{
INVESTIGACIONES
}

\section{¿Influye la universidad en la empleabilidad exitosa de los titulados de pedagogía?}

\author{
Does the university influence the successful employability \\ of graduates from teaching programs?

\section{Gonzalo Cifuentes Gómez $z^{a}$, Mari Carmen Villarroel Farías ${ }^{b}$, Orietta Geeregat Vera ${ }^{c}$}

\author{
Universidad de Santiago de Chile. \\ Correo electrónico: gonzalo.cifuentes@usach.cl \\ Universidad de La Frontera. mari. \\ Correo electrónico: villarroel@ufrontera.cl. \\ Universidad de La Frontera. \\ Correo electrónico: orietta.geeregat@ufrontera.cl.
}

\begin{abstract}
RESUMEN
El estudio exploró en titulados de Pedagogía, los factores externos e internos que influyen en su empleabilidad. Utilizando una aproximación metodológica mixta, el estudio consideró la aplicación de un cuestionario de empleabilidad y autoeficacia pedagógica a 59 titulados de las carreras de pedagogía en enseñanza media y el desarrollo de tres entrevistas en profundidad y un grupo focal. Respecto a los conocimientos de los titulados, los resultados muestran que le atribuyen una mayor importancia en su formación pedagógica a la experiencia laboral por sobre la formación recibida en la universidad, y que consideran que su formación disciplinar fue más sólida que la pedagógica. Paralelamente, los titulados atribuyen gran importancia a las redes que generaron en pregrado para obtener el primer trabajo, así como a sus atributos personales (compromiso, responsabilidad, proactividad) para mantenerse en ellos, agregan, que estos atributos sumados a la experiencia, les facilitan poder alcanzar cargos de mayor responsabilidad.
\end{abstract}

Palabras claves: Formación inicial de profesores, empleabilidad, factores externos, factores internos.

\section{ABSTRACT}

This study explored the external and internal factors that influence the employability of graduates from teaching programs. Using a mixed methodological approach, this study involved the application of an employability and pedagogical self-efficacy questionnaire and the development of three in-depth interviews as well as a focus group of 59 graduates from teaching programs for secondary education. Regarding graduates' professional knowledge, the results show that in terms of their pedagogical training they consider their work experience more important than the training they received in university. They also consider that university education in the subject area was more robust than in the pedagogical area. At the same time, graduates attribute great importance to the networks generated during undergraduate studies in securing their first jobs, as well as to their personal attributes (commitment, responsibility, proactivity) in keeping these jobs. Furthermore, they add that these attributes, together with the experience, provide them with opportunities to access positions of greater responsibility.

Keywords: Initial teacher training, Employability, External factors, Internal factors. 


\section{PROBLEMA DE INVESTIGACIÓN}

En las últimas décadas en Chile la autoridad central ha manifestado interés por la Formación Inicial Docente (FID), siendo uno de sus últimos hitos la promulgación de la ley 20.903 en el año 2016, que crea el Sistema de Desarrollo Profesional Docente. Dicha ley aumenta las exigencias de ingreso a las carreras de pedagogía, haciendo obligatoria la acreditación de las carreras e impulsando la aplicación de evaluaciones diagnósticas y de seguimiento en los resultados de formación de sus estudiantes. Además, le otorga especial importancia a la formación integral asociado a la inclusión, diversidad, ciudadanía, prácticas tempranas y la didáctica de la especialidad, cautelando de esta manera la calidad de la formación de sus titulados.

Sumado a lo anterior, diversos reportes e investigaciones (Avalos 2009; OECD 2004; Cox 2007; Corvalán 2010; Mineduc 2005, Ruffinelli 2013) surgidos a partir de la experiencia acumulada en la década de los 90', dan cuenta de una serie de problemas estructurales en la formación de profesores, los que traen como consecuencia la falta de conocimiento práctico por parte de los estudiantes de pedagogía, la que se debe, entre otras causas, a la separación entre la formación universitaria recibida por los profesores en formación y el contexto escolar que constituirá su ámbito de práctica futura.

Los problemas descritos se dan en el contexto de un mercado de carreras de pedagogía que hasta la promulgación de la ley de carrera docente el año 2016, se encontraba mínimamente regulado y marcado por el dinamismo de nuevas instituciones privadas capaces de ofertar nuevos programas y alternativas (Cox, Meckes y Bascopé 2010). En este escenario, evidentemente la calidad de los programas es profundamente dispar, caracterizándose por su heterogeneidad tanto en términos de articulación de mallas curriculares como en sus requisitos de selección.

Paralelamente, la sociedad demanda de las nuevas generaciones de profesores requerimientos más amplios y complejos que hace unas décadas atrás. Los futuros profesores deben estar preparados para trabajar con contenidos de mayor nivel, ya no basta el conocimiento factual, sino que debe ser un profesional capaz de crear y orquestar ambientes de aprendizaje complejos, involucrando a los estudiantes en actividades apropiadas, de manera que éstos puedan construir su propia comprensión del contenido (Bransford, Brown, y Cocking 2000; Bustos y Coll 2010; Cox, Beca y Cerri 2011; Darling-Hammond, y Bransford 2005; Haydn y Barton 2007; Walsh 2005). En este sentido, el proceso de formación de profesores debe ser una práctica educativa que potencie cambios cualitativos en el desarrollo y en el aprendizaje de sujetos que desean prepararse profesionalmente para enseñar (Libaneo 2008).

En este escenario, adentrase en el grupo de profesores recién titulados pareciera aportar importantes antecedentes para la discusión. Explorar las formas que utilizan para insertarse y mantenerse de manera exitosa en el mundo laboral, así como la valoración que hacen de su proceso de formación universitario, es de vital importancia para orientar y proponer mejorar a la formación inicial docente.

\section{FACTORES QUE INFLUYEN EN LA EMPLEABILIDAD DE LOS PROFESORES NÓVELES}

El tránsito desde el egreso de la Universidad y la iniciación en el mundo laboral suele pensarse como un proceso lineal, sin embargo, lejos de ello el mercado laboral tiene características variadas y diversas en donde intervienen una serie de factores objetivos, 
subjetivos, personales y contextuales que resultan difíciles de anticipar y controlar (Rojas, Chávez y Suazo 2011).

Para el profesor, el tránsito hacia el mundo laboral se torna más dificultoso si se considera el bajo impacto que tiene la FID en los recién titulados (Ávalos, Carlson y Aylwin 2004; Manzi 2010; MINEDUC 2005; OCDE 2004). Dicho impacto se expresa en las primeras aproximaciones en el aula, en donde el profesor novel tiende a refugiarse en su experiencia como estudiante, a partir de la cual imita las prácticas pedagógicas de sus profesores o bien, se somete a las demandas de su contexto laboral (Latorre 2005), para lo cual deja de lado los conocimientos adquiridos en su formación universitaria.

Resulta evidente que un recién titulado posee un nivel de competencias que se asocia a las de un novato en su profesión. Al respecto Corvalán señala que para alcanzar un mayor nivel pueden llegar a transcurrir entre cinco y siete años de práctica profesional activa (Corvalán 2010). En este periodo, Cornejo reconoce dos fases; la primera, denominada "umbral o antesala" que se extiende entre el primer y el sexto mes de docencia; la segunda, denominada "madurez y crecimiento" se extiende entre los tres y cinco años de ejercicio de la profesión (Cornejo 1999).

Para Vonk la etapa inicial de la profesión docente constituye un proceso de inducción que se compone de dos momentos claves: "ambientación” y "progresión”, en donde el recién titulado debe asumir que está en una transición desde un "sujeto en formación" hasta la de un "profesional autodirigido" (Vonk 1996). Finalmente, Imbernón entiende esta etapa como un proceso de "socialización" en la que el profesor recién titulado debe integrarse como miembro activo y participante del colectivo profesional (Imbernón 2007).

A partir de la revisión de la literatura nacional (Ávalos 2009; Ávalos, Carlson y Aylwin 2004; Cabezas, Gallego, Santelices y Zarhi 2011; Meckes y Bascopé 2010; MIDE-UC 2008; Ruffinelli y Guerrero 2009), es posible identificar una serie de factores más o menos comunes que inciden en los profesores nóveles al momento de encontrar su primer trabajo y mantenerse en él. El esquema 1 resume los distintos factores involucrados en la medición de la empleabilidad.

Esquema 1. Factores que influyen en la medición del constructo empleabilidad

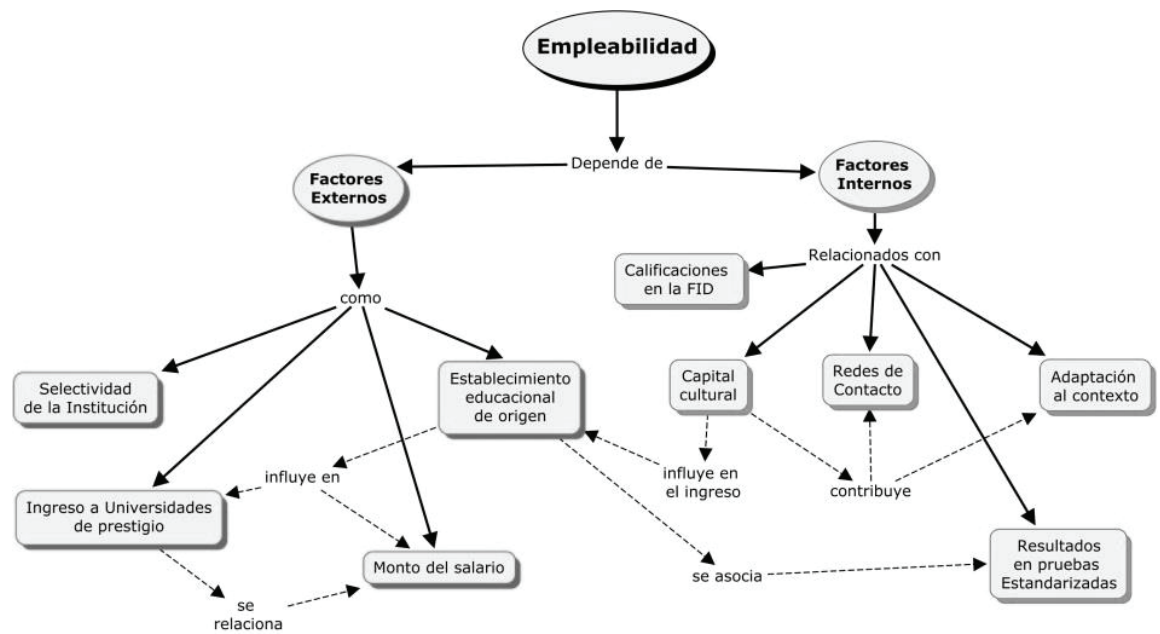

Fuente: Geeregat, Cifuentes y Villarroel (2016). 
El esquema organiza los factores descritos por la literatura nacional en dos grandes grupos. Por una parte están los factores externos al individuo que de igual manera influyen en la obtención de un empleo. Así por ejemplo encontramos como el prestigio y selectividad de la universidad en donde el profesor se formó o el establecimiento educacional de origen, son factores que se han reportado como influyentes al momento de obtener un empleo. En paralelo se incluye el factor salarial, el cual a juicio de Meckes y Bascopé, estaría influyendo en la decisión de un profesor recién egresado al momento de aceptar un trabajo (Meckes y Bascopé 2010).

El otro grupo de factores denominado internos, se relacionan con las características particulares de los sujetos. Aquí podemos encontrar desde elementos más tangibles como las calificaciones obtenidas durante el proceso de formación universitaria o los resultados en la prueba de selección universitaria, hasta factores menos tangibles tales como las redes de contacto, el capital cultural y la adaptación al contexto que logra el profesor novel durante el primer año de ejercicio de la profesión.

\section{OBJETIVO}

Explorar en los factores externos e internos a los titulados de pedagogía que influyen en su empleabilidad durante el primer año de ejercicio de la profesión.

\section{MÉTODO}

Por medio de una aproximación mixta (Hernández, Fernández y Baptista 2010), el estudio consideró en primer lugar la aplicación de un cuestionario que observó empleabilidad y autoeficacia pedagógica en egresados de pedagogía. Los resultados obtenidos orientaron el desarrollo de tres entrevistas en profundidad y un grupo focal, cuyo propósito se centró en complementar los resultados cuantitativos, profundizando en las experiencias laborales de los participantes.

\subsection{FASE CUANTITATIVA}

\section{a) Validez y Confiabilidad}

El cuestionario de autopercepción de conocimiento pedagógico se compone de 20 ítems organizados en torno a dos dimensiones Autopercepción Conocimiento Pedagógico (ACP) y Fuente del Conocimiento Pedagógico (FCP). Respecto a su confiabilidad, el instrumento presenta un Alfa de Cronbach de 0,819. Al analizar por dimensiones, los valores se mantienen por sobre un 0.8 ( 0,804 para ACP y 0,854 para FCP), lo que a juicio de Hogan daría cuenta de una alta consistencia interna (Hogan 2004). Finalmente, al hacer un análisis por ítems, es posible observar que no existen variaciones sustantivas en la confiabilidad.

La validez de contenido del instrumento se cauteló a través del juicio de expertos, en donde tres académicos, con experiencia en formación docente, revisaron la pertinencia de los ítems. Para ello se utilizó como criterio para eliminar, modificar o mantener un $80 \%$ 
de acuerdo interjuez (AERA, APA y NCME 2014). Los resultados obtenidos permitieron mejorar la redacción de algunos ítems del cuestionario.

Finalmente, considerando las limitaciones que impone el tamaño de la muestra (Pérez y Mendrano 2010) se optó por evaluar la validez de constructo por medio del cálculo de correlaciones entre los ítems y sus dimensiones asociadas. Los resultados muestran que la dimensión ACP presenta correlaciones altas y significativa $(r>0,375 ; \mathrm{p}<0,05)$ con todos los ítems que la componen. Por su parte, al correlacionar la dimensión ACP con los ítems de la dimensión FCP, se presentan correlaciones bajas y no significativas $(r<0,194$; $\mathrm{p}>0,179)$.

Respecto a la dimensión FCP, los resultados muestras correlaciones altas y significativas ( $r>0,485 ; p<0,001)$ entre la dimensión y sus ítems. Asimismo, las correlaciones entre los ítems de FCP y la dimensión ACP, muestra correlaciones bajas y no significativas $(\mathrm{r}<0,194 ; \mathrm{p}>0,088)$, a excepción del ítem $1(\mathrm{r}=0,325 ; \mathrm{p}<0,05)$.

En consideración de los antecedentes expuestos, es posible concluir que el cuestionario aplicado posee criterios de validez y confiabilidad suficientes para utilizar sus resultados en los propósitos definidos en la investigación.

\subsection{FASE CUALITATIVA. ENTREVISTAS Y GRUPOS FOCALES}

Desde la perspectiva de Creswell, la fase cualitativa implementada se enmarca en un estudio de tipo Fenomenológico (Creswell 2013). En particular este tipo de aproximación no pretende establecer una explicación causal del fenómeno, sino que describe el significado de las experiencias vividas por un grupo de personas, resaltando los aspectos individuales y subjetivos de la experiencia.

\subsection{CRITERIOS DE RIGOR}

El estudio consideró entre los criterios de rigor propuestos por Mendizábal, el resguardo de la credibilidad de la investigación y de la seguridad en la obtención de los datos (Mendizábal 2006).

Respecto de la credibilidad se resguardo en particular el criterio de "Obtención de datos ricos" por medio de tres entrevistas extensas y un grupo focal que se tradujeron en 52 páginas de transcripciones, a partir de las cuales se realizó la codificación de las respuestas de las entrevistadas. Durante las entrevistas se tuvo en consideración profundizar en los temas de interés, a través de la formulación de preguntas que originalmente no estaban consideradas en la pauta.

Respecto del criterio de Seguridad, los datos de las participantes fueron recopilados siguiendo un mismo procedimiento, vale decir, se contactó preliminarmente a las entrevistadas y se les explicó el propósito de la investigación. Por otra parte se les solicitó previamente autorización para grabar en audio de la conversación, se cauteló realizarla en el horario que más les acomodara y finalmente las entrevistas y grupo focal se desarrolló en lugares que no tuvieran distracciones tales como teléfonos o alto tránsito de personas. Las precauciones antes descritas contribuyeron en el Rapport con las entrevistadas, lo que ciertamente facilitó el proceso de recolección de información y la profundidad a la que se puede acceder en determinados temas. 


\subsection{SUJETOS DE ESTUDIO, TÉCNICAS DE PRODUCCIÓN Y ANÁLISIS DE DATOS}

La recopilación de datos se realizó en la región de los Lagos y La Araucanía, con titulados de las carreras de Pedagogía en Castellano, Matemática e Historia de La Universidad de la Frontera, entre los años 2010 y 2015. Las seis participantes son mujeres con rangos de edades entre los 24 y 27 años, todas con más de un año de experiencia en el ejercicio docente.

En las entrevistas y grupos focales se utilizó una pauta compuesta por siete preguntas, las que profundizaron en los siguientes temas:

a) Contexto: compuesto de una pregunta que buscaba situar paulatinamente al entrevistado en el tema de interés.

b) Búsqueda del primer empleo: compuesto de dos preguntas que buscaban conocer las estrategias que utilizaron las entrevistadas para encontrar su primer trabajo y el tiempo que les tomó encontrarlo.

c) Permanencia en el trabajo: por medio de tres preguntas se buscaba profundizar en la experiencia de las entrevistadas respecto a los factores que influyen en permanecer en el trabajo; asimismo se les consultó respecto a los aspectos en que se sintieron más y menos preparadas por la Universidad.

d) Cierre: Finalmente se dejó una última pregunta para que pudiesen recomendar, desde su perspectiva, los temas que deberían ser mejorados en la formación de pregrado de la universidad.

Cabe señalar que la entrevista incluía la lectura y firma del consentimiento informado, en donde se exponían los propósitos de la investigación.

El proceso de análisis se realizó utilizando la transcripción literal del audio de las entrevistas, las cuales fueron cargadas en el programa Atlas Ti 7.5.4. Respecto de la codificación se partió con una de tipo abierta, para posteriormente identificar categorías. La agrupación de estos códigos se realizó considerando el contexto, condiciones intervinientes y consecuencias (Strauss, Corbin y Zimmerman 2002), elaborando así las familias de códigos.

\section{RESULTADOS FASE CUANTITATIVA}

\subsection{CARACTERIZACIÓN DE LA MUESTRA}

El estudio cuantitativo se compuso de 59 titulados de Pedagogía, distribuidos en 24 profesores de Castellano (40,7\% del total), 6 de Matemática (10,2\%), 27 de Historia $\left(45,8 \%\right.$ del total) y 1 de Educación Física $(1,7 \%)^{1}$, quienes egresaron entre los años 2007 y 2014, con una mayor concentración del 2009 en adelante ( $88 \%$ del total).

Respecto a la dependencia de los establecimientos tanto de educación básica como de media, los resultados de diferencia de medias $\left(\mathrm{X}^{2}=5,601 \mathrm{gl}=4 \mathrm{p}>0,05\right)$ muestran que no existen diferencias significativas. La tabla 1 muestra en detalle dichos resultados.

Para efectos de los análisis inferenciales que se presentan más adelante, el profesor de educación física fue eliminado y solo se trabaja en base a los resultados de las otras tres carreras. 
Tabla 1. Distribución por dependencia

\begin{tabular}{|l|c|c|}
\hline \multicolumn{1}{|c|}{ Dependencia } & \% Ed. Básica & \% Ed. Media \\
\hline Municipal & $48,3 \%$ & $44,8 \%$ \\
\hline Particular Subvencionado & $48,3 \%$ & $50 \%$ \\
\hline Privado & $3,4 \%$ & $0 \%$ \\
\hline Administración delegada & 0 & $5,2 \%$ \\
\hline Total & $\mathbf{1 0 0 \%}$ & $\mathbf{1 0 0 \%}$ \\
\hline
\end{tabular}

Respecto a las comunas de origen de los establecimientos, es posible observar que tanto para enseñanza básica como media, predomina la comuna de Temuco, 33,9\% y 45,8\% respectivamente. En esta misma dimensión fue posible observar que tanto para educación básica como para educación media más del $88 \%$ de los titulados cursó sus estudios en un contexto urbano, no detectándose diferencias significativas entre niveles educativos $\left(\mathrm{X}^{2}=199 \mathrm{gl}=1 \mathrm{p}>0,05\right)$.

\subsection{ANTECEDENTES ACADÉMICOS}

Respecto a la preferencia por la carrera de Pedagogía, es posible observar el 83\% (49) de los titulados declara haber postulado en primera preferencia, un $11,9 \%$ (7) en segunda y un $5,1 \%$ (3) en tercera.

Una vez titulados, el 33,9\% (20) señalar haber realizado algún curso de perfeccionamiento a través del Centro de Perfeccionamiento, Experimentación e Investigaciones Pedagógicas (CPEIP), del Ministerio de Educación (MINEDUC), de manera presencial y en universidades del Consejo de Rectores de Chile (CRUCH).

Respecto a la formación de postgrado, el 27\% (16) indica estar cursando o haber finalizado un Magíster, en áreas como currículum, evaluación, didáctica, gestión y administración educativa, así como en temas propios de las disciplinas de origen de los titulados, tales como Ciencias Sociales Aplicadas, Estadística y Didáctica de la Lengua y la Literatura, principalmente en universidades del CRUCH (75\%).

En cuanto a la participación de los titulados en algún proceso de evaluación del MINEDUC, como por ejemplo Evaluación Docente, AEP o AVDI, a la fecha, solo el $15,3 \%$ (9) ha participado.

\subsection{ANTECEDENTES LABORALES}

Respecto a sus antecedentes laborales, el 79,7\% (47) de los titulados que participaron del estudio indican haber encontrado su primer trabajo como profesor en menos de 6 meses. En relación a la dependencia administrativa de los establecimientos educacionales en que se desempeñan, el 47,5\% (28) de los titulados lo hace en un establecimiento particular subvencionado, el 16,9\% (10) en uno municipal y el 15,3\% (9) en uno privado. Cabe señalar que el 18,6\% (11) de los titulados declara trabajar en Educación Superior. 
En cuanto a su permanencia en sus actuales trabajos, el 64,5\% declara que llevan entre menos de un año y tres años. Respecto a las horas de contrato el $61 \%$ de los entrevistados trabaja más de 34 horas a la semana, mientras que el 27,1\% lo hace en un rango que va entre las 23 y 33 horas a la semana.

Finalmente, respecto al tipo de trabajo que realizan en los establecimientos educacionales, el 76,3\% lo hace como profesor de aula y solo un 5,1\% ejerce labores en la Coordinación Académica. En cuanto a los niveles en los que han realizado clases, los titulados se distribuyen homogéneamente entre 5to y 6to básico (30,5\%), 7mo y 8 avo básico $(32,2 \%)$ y 1ero a 4 to medio $(28,8 \%)$. Adicionalmente, algunos titulados señalan que también han realizado docencia en Preuniversitarios, Educación de Adultos y Educación Superior.

\subsection{AUTOPERCEPCIÓN CONOCIMIENTO PEDAGÓGICO Y FUENTE DEL CONOCIMIENTO PEDAGÓGICO}

Cuando se les pregunta a los titulados $(\mathrm{N}=59)$ respecto a su conocimiento pedagógico (en base a los estándares orientadores para carreras de Pedagogía del MINEDUC) estos presentan altos grados de autoconfianza, con medias superiores a 4 ("De acuerdo"), a lo que se agrega una baja dispersión de los datos ( $\sigma<0,748$ a excepción de la pregunta 8). La tabla 2 muestra un detalle por pregunta.

Tabla 2. Detalle por pregunta Conocimiento Pedagógico

\begin{tabular}{|l|c|c|c|c|c|c|c|c|c|c|}
\hline Ítems & CP_1 & CP_2 & CP_3 & CP_4 & CP_5 & CP_6 & CP_7 & CP_8 & CP_9 & CP_10 \\
\hline Media & 4,10 & 4,10 & 4,23 & 4,24 & 4,41 & 4,03 & 4,14 & 3,71 & 4,58 & 4,50 \\
\hline Mediana & 4,00 & 4,00 & 4,00 & 4,00 & 4,00 & 4,00 & 4,00 & 4,00 & 5,00 & 5,00 \\
\hline Moda & 4 & 4 & 4 & 4 & 4 & 4 & 4 & 4 & 5 & 5 \\
\hline Desv. Estándar &, 484 &, 742 &, 655 &, 625 &, 591 &, 748 &, 611 & 1,108 &, 596 &, 714 \\
\hline Mínimo & 3 & 2 & 2 & 2 & 3 & 2 & 2 & 1 & 2 & 2 \\
\hline Máximo & 5 & 5 & 5 & 5 & 5 & 5 & 5 & 5 & 5 & 5 \\
\hline
\end{tabular}

Cuando se les consulta a los profesores $(\mathrm{N}=59)$ por la fuente de los conocimientos pedagógicos adquiridos, en una escala de 1 a 5 , donde uno es la Universidad y 5 su experiencia laboral, sus opiniones están más próximas a su experiencia laboral con medias superiores a 3. La excepción la constituye la pregunta "Sé comunicarme de manera efectiva oralmente y por escrito en diversas situaciones asociadas a mi quehacer docente." ( $\bar{X}=2,91)$, en donde le atribuyen un mayor aporte a la Universidad. Caso contrario lo constituye la pregunta "Conozco la cultura escolar", en donde atribuyen su conocimiento a la experiencia laboral. La tabla 3 muestra el detalle de los resultados. 
Tabla 3. Detalle por pregunta Fuente Conocimiento Pedagógico

\begin{tabular}{|l|c|c|c|c|c|c|c|c|c|c|}
\hline \multicolumn{1}{|c|}{ Ítems } & FCP_1 & FCP_2 & FCP_3 & FCP_4 & FCP_5 & FCP_6 & FCP_7 & FCP_8 & FCP_9 & FCP_10 \\
\hline Media & 3,98 & 3,68 & 3,33 & 3,00 & 3,63 & 3,16 & 4,39 & 4,11 & 2,91 & 3,25 \\
\hline Mediana & 5,00 & 4,00 & 4,00 & 3,00 & 4,00 & 3,00 & 5,00 & 5,00 & 3,00 & 3,00 \\
\hline Moda & 5 & 5 & 5 & 3 & 5 & 5 & 5 & 5 & 3 & $3^{\text {a }}$ \\
\hline Desv. estándar & 1,261 & 1,454 & 1,466 & 1,389 & 1,345 & 1,512 &, 856 & 1,138 & 1,456 & 1,399 \\
\hline Mínimo & 1 & 1 & 1 & 1 & 1 & 1 & 2 & 1 & 1 & 1 \\
\hline Máximo & 5 & 5 & 5 & 5 & 5 & 5 & 5 & 5 & 5 & 5 \\
\hline
\end{tabular}

a. Existen varias modas. Se muestra el menor de los valores

\subsection{ASOCIACIONES ENTRE VARIABLES}

De acuerdo a lo planteado en el marco referencial, era plausible explorar asociaciones entre el conocimiento pedagógico y la fuente de dicho conocimiento, entendido esto como autoeficacia, con algunas de las variables que observaron la empleabilidad de los profesores. Los resultados muestran que al correlacionar el nivel de conocimiento declarado por los profesores respecto a variables asociadas al trabajo como por ejemplo Permanencia, Horas de contrato y Tiempo que se demoró en obtener primer trabajo es posible observar una asociación directa positiva con el número de horas de contrato $(r=0,292 ; p<0,05)$, por tanto, a mayor cantidad de horas de contrato, los profesores presentan mayor confianza en sus conocimientos pedagógicos.

Lo anterior es importante, en el sentido que si bien dicha autoconfianza parece estar influyendo a la hora de permanecer en un trabajo, ésta es atribuida en mayor medida a los conocimientos que adquieren los profesores en el ejercicio mismo de la profesión y no en su paso por la Universidad.

Por su parte, al correlacionar nuevamente la Permanencia, Horas contratadas y Tiempo en obtener primer trabajo, utilizando como variable dependiente Fuente del Conocimiento Pedagógico, es posible encontrar una fuerte relación lineal positiva con la variable Permanencia $(r=0,420 ; p<0,01)$. Este dato, vuelve a ratificar la poca injerencia que le atribuyen los profesores en ejercicio a la formación pedagógica recibida en la Universidad. Dicho resultado estaría indicando que aquellos profesores que consideran que la fuente de su conocimiento pedagógico se construyó en el ejercicio de su profesión, son precisamente los que han permanecido más tiempo en sus actuales empleos. La tabla 4 muestra en detalle los resultados. 
Tabla 4. Correlación de Conocimiento Pedagógico y Fuente del conocimiento Pedagógico con variables independientes Permanencia, Horas de contrato y Tiempo en encontrar primer trabajo.

\begin{tabular}{|c|c|c|c|}
\hline VI / VD & Permanencia & $\begin{array}{c}\text { Horas de } \\
\text { contrato }\end{array}$ & $\begin{array}{c}\text { Tiempo en encontrar } \\
\text { primer trabajo }\end{array}$ \\
\hline $\begin{array}{c}\text { Conocimiento } \\
\text { Pedagógico }\end{array}$ & $-0,178$ & $0,292 *$ & 0,082 \\
\hline $\begin{array}{c}\text { Fuente del conocimiento } \\
\text { Pedagógico }\end{array}$ & $0,420 * *$ & 0,042 & 0,200 \\
\hline
\end{tabular}

Significancias: $* * *<0,001 * *<0,01 *<0,05$

\section{RESULTADOS FASE CUALITATIVA}

Las entrevistas en profundidad y el grupo focal desarrollado a docentes egresados de la Universidad de La Frontera se enfocaron en profundizar en los factores que influyen en la obtención del primer trabajo, así como en la permanencia en éste. Adicionalmente y a partir de los resultados obtenidos en la fase cuantitativa, se profundizó la percepción de las tituladas respecto a la fuente principal de origen de sus conocimientos en el ámbito disciplinar y pedagógico.

Los datos proporcionados durante la aplicación de esta técnica, dieron lugar a $\underline{7}$ familias de códigos, que son expuestas a continuación:

Respecto a las "Exigencias del establecimiento hacia la labor docente", las entrevistadas dan cuenta que en su primer año se les exige en mayor medida responsabilidad, proactividad y compromiso con la institución, el que se refleja en el desarrollo de actividades fuera del horario laboral. Adicionalmente, coinciden en que se espera de ellas un sólido dominio de grupo, lo que les ayuda a no ser "mal vistos" por el resto de los profesores de los establecimientos.

"yo llegué [y me dijeron] "esperamos tu compromiso con el colegio, que te pongas la camiseta" cosas así "que puedas aportar ideas nuevas" y de hecho como que cada profesor como que busca eso como que puedo proponer nuevo" (mujer, 2 años de titulada).

Respecto a la familia de códigos "Implicancias para encontrar el primer trabajo", la experiencia de las docentes entrevistadas indica que tanto ellas como sus compañeros tienden a encontrar su primer trabajo a través de dos mecanismos. El primero es a través de una búsqueda individual, que consiste principalmente en la entrega de su currículum sin conocer el lugar donde postulan; el segundo mecanismo se refiere al uso de las redes de contacto profesional que lograron formar durante su formación de pregrado.

Así, si un compañero está trabajando, comienza a recomendar a sus compañeros (as) y a compartir información en caso de existir una vacante.

"no y eso es súper potente porque a mí me han llegado un montón de datos este año como de "oye necesitan profe de lenguaje en tal parte si quieres completar horario y 
la cuestión" y son puros compañeros que salieron años anteriores o que están en mí mismo nivel en otros liceos o en otras ciudades incluso" (Mujer, 1 año de titulada).

Por su parte, existe un grupo de estudiantes que son contratados en los mismos establecimientos en donde realizaron sus prácticas profesionales ${ }^{2}$, dado el buen desempeño que alcanzaron en ella.

Otra forma utilizada por los titulados para encontrar su primer trabajo lo denominaremos topográfica, la cual alude a que la contratación se genera porque el titulado es ex alumno del establecimiento o porque creció en esa comuna o sector. Dichos casos se dan principalmente en comunas de menor tamaño tales como Gorbea, Pitrufquén, Nueva Imperial, así como en Chiloé.

Esta última forma es particularmente interesante, dado que, independiente de la trayectoria formativa del titulado, influyen la idiosincrasia, la empatía o familiaridad, cuestiones todas, que no dependen los factores clásicos reportados por la literatura, tales como el prestigio de la institución de formación. Este retorno topográfico y humano debe considerarse como un aspecto fundamental y determinante por el compromiso de cambio social que tiene todo proyecto educativo.

Una vez superada la barrera del ingreso al mundo laboral, se consultó respecto a los factores que a su juicio influyen tanto en mantener el trabajo, como en ser valorados en dicho contexto. En particular, fue posible agrupar una familia de códigos denominada "Factores que inciden en la renovación de contrato"; estos dan cuenta que son variados los componentes que confluyen en ello. Por un lado, se afirma que son relevantes las actividades de enseñanza que desarrollan los docentes recién titulados durante el primer año, pues se espera que estas incorporen estrategias distintas a las utilizadas en los establecimientos tradicionalmente. No obstante, es posible deducir del relato de las entrevistadas, que no tienen mayor claridad respecto al cambio cualitativo que producen sus estrategias de enseñanza ni del valor emergente de éstas.

Paralelamente, fue posible observar que en los establecimientos existe una gran valoración al dominio de contenido de los docentes recién egresados, así como a la planificación de sus clases. Desde sus experiencias, existe coincidencia respecto a la mayor supervisión que vivieron de parte de los docentes directivos de los establecimientos durante el primer año laboral, los que incluso recurren a la opinión de los estudiantes para conocer el desempeño de los docentes.

"son súper exigentes respecto a los profesores, cuando tú vas y no llevas una clase preparada ellos lo notan enseguida y lo dicen, entonces tiene que ver mucho eso de como uno se para frente a ellos, como respondes sus dudas, ellos se dan cuenta cuando no sabes y no respondes. Tiene que ver eso como si uno está al nivel de las exigencias de los propios estudiantes igual" (Mujer, 1 año de titulada).

Otro de los factores que influye en la renovación del contrato corresponde a la relación que se da entre pares, estudiantes y directivos del establecimiento. A juicio de las entrevistadas, en el primer año es importante la relación que generan particularmente con los pares, siendo bien valorado aportar en la generación de un apropiado ambiente laboral.

La práctica profesional integrada en la Universidad de La Frontera, tiene una duración de 10 meses con una jornada de 22 horas semanales. 
"el buen trato con tus colegas también, porque hay muchos que no se llevan bien y simplemente los sacan porque no generan un buen ambiente laboral” (Mujer, 5 años de titulada).

Respecto a la familia de códigos "Aspectos pedagógicos y disciplinares aprendidos en el campo laboral", fue posible observar que en el ámbito disciplinar existen pocas falencias. Si bien señalan que algunas veces se vieron en la necesidad de revisar el contenido, este ámbito no constituyó un problema fundamental en su desempeño laboral en el primer año. No obstante, desde el ámbito pedagógico, los titulados manifiestan que tuvieron ciertas falencias una vez insertos en el sistema laboral, los van desde completar un libro de clases, enfrentar una jefatura o planificar una determinada unidad.

"la verdad yo no sabía planificar, me di cuenta después cuando tenía que armar mis planificaciones, modelos $T$ y todo un tema que hay detrás... al final tienes que aprender igual el tema de la jefatura, yo no tuve jefatura integrada en la malla entonces al final los papás llegan con los niños y como que quieren que les soluciones la vida, entonces es complicado." (Mujer, 2 años de titulada).

En esta línea, las entrevistadas coinciden en que uno de los principales desafíos que les impuso el contexto laboral se relacionó con su escaso conocimiento en temas ligados a la orientación, así como al desconocimiento que tenían de la normativa educacional frente a temas de drogadicción. Paralelamente demandan de su formación inicial un mayor énfasis en las leyes laborales que rigen a los docentes del país.

Otro de los aspectos analizados es la percepción que tienen de las "Herramientas entregadas por la Universidad". Los códigos señalan que el prestigio de la casa de estudios en donde se formaron, les da seguridad al momento de enfrentar el mundo laboral. Del mismo modo, se afirma que la preparación disciplinar es muy buena, rara vez, existen problemas para entender el contenido y generar aprendizaje significativo en los estudiantes. Atribuyen esta fortaleza a la calidad y preparación de los académicos de la universidad.

Asimismo, las entrevistadas coinciden que las actividades que realizaron en forma paralela a su formación curricular, tales como ser ayudante, hacer reemplazos o participar en agrupaciones estudiantiles, les brindó importantes herramientas para enfrentar el mundo laboral.

"si, hice muchas agrupaciones, qué loco, eso igual me sirvió. Una agrupación donde hacemos clases, fuimos a varios colegios a trabajar y ahí uno igual va aprendiendo, quizás en ese momento uno no sabe todas las cosas pero después uno ya sabe" (Mujer, 1 año de egresada).

En esta línea, el relato de las entrevistadas da cuenta que la universidad les brindó distintas oportunidades para desarrollarse profesionalmente, ya sea en talleres, ayudantías o actividades de vinculación temprana con el ejercicio de la profesión. En este caso, las entrevistadas manifiestan que esta posibilidad es un eje muy relevante para la profesión, porque estas condiciones les permiten aprender a exponer en público o perfeccionarse en dominio de grupo, por ejemplo. 
En cuanto a la familia de códigos "Aspectos que debe mejorar la Universidad en la formación de profesores", la experiencia de las tituladas da cuenta que se debe poner mayor énfasis en las didácticas de especialidad, así como generar los espacios para que los estudiantes puedan aproximarse de mejor forma a la realidad educacional regional y del país.

"yo creo que eso falta también aquí en la Universidad, que alguien te enseñe que en el fondo los chicos se van a acercar a ti te van a decir "profe me siento mal, tengo tal problema, ¿qué puedo hacer?” (Mujer, 5 años de titulada).

Por otro lado, los códigos expresan que los docentes deben generar vinculaciones con lo pedagógico y no solo generar sus estrategias bajo el alero del aspecto teórico y disciplinario. En este mismo contexto, las entrevistadas manifiestan que un buen acercamiento a la didáctica sería la implementación de prácticas tempranas. En este caso, que los profesores en formación tengan la oportunidad de observar una situación real de enseñanza. Y del mismo modo, conocer cómo se puede estructurar una reunión de apoderados o una entrevista personal con un estudiante.

“reforzar lo del profe jefe, es súper importante, no sé... saber pararte en una reunión de apoderados, en una entrevista con un niño, con un apoderado porque tampoco se puede decir cosas muy chocantes porque no sabes cómo van a reaccionar" (Mujer, 2 años de titulada).

Otro punto relevante que manifiestan las entrevistadas corresponde a la implementación de clases sobre el proyecto de integración escolar. En este aspecto afirman que la universidad no entrega herramientas para tratar con estudiantes que poseen asperger, déficit atencional o diversas complejidades del aprendizaje. Aspectos que siendo relevantes, es necesario dosificar en una formación inicial de profesores.

La familia de códigos "Proactividad y responsabilidad, cualidades relevantes para la docencia", dio cuenta que desde la experiencia de las entrevistadas, el sentido de la responsabilidad constituye un aspecto muy valorado por los establecimientos y que en paralelo, es un ámbito que debe ser trabajado por la institución. Si bien reconocen que ésta constituye una competencia más de carácter individual, creen que la universidad debería intencionarla mejor a lo largo de la carrera.

En esta línea, las entrevistadas atribuyen un importante peso a la práctica profesional, pues ahí tienen la posibilidad de poner en juego la formación disciplinar y pedagógica recibida durante su formación, así como aspectos individuales, tales como la proactividad o la responsabilidad.

"el profesor de práctica nos tenía corriendo toda la semana, pero toda la semana y si tu no le cumplías era un caracho pero del porte de un buque...entonces eso nos obligó a ser mucho más metódicas y más responsables y al final se nos quedó, nosotras no éramos tan responsables" (Mujer, 1 año de titulada).

Profundizando, las entrevistadas consideran que aquí un rol fundamental lo juegan los docentes tutores de los establecimientos. A su juicio, de ellos depende en gran medida el éxito de la práctica profesional como una instancia real de aprendizaje. 
"Sí, respecto a eso en la práctica no todos tienen la suerte que tuve yo de tener un profe que compartiera allá, si me dijeran si aprendí de un profe yo les diría que no, yo no aprendí de ningún profe porque mis profes no eran buenos entonces como que eso igual es importante saber que profes van a ser tus profes guías y como son tus profes guías" (Mujer, 2 años de titulada).

En ese sentido, la formación universitaria debería intentar cautelar de la mejor forma posible, la elección de los docentes tutores, de manera que constituyan una guía para los profesores en formación.

Lo expuesto hasta aquí, da cuenta de las 7 familias de códigos obtenidos en las entrevistas, sin embargo, existieron otros hallazgos no descritos en esta oportunidad, ya que se consideró que estos eran los pertinentes para dar respuesta al objeto de estudio.

\section{DISCUSIÓN Y CONCLUSIONES}

La literatura coincide en señalar que la empleabilidad se asocia a factores tanto internos como externos al individuo. No obstante, estos factores tienden a ser vistos de manera independiente, lo que dificulta la observación de relaciones que podrían brindar una compresión más contextualizada del fenómeno. Analizar el constructo como un conjunto de factores internos y externos interrelacionados permite abrir la forma de aproximarnos al fenómeno de estudio y establecer relaciones teóricas entre unos y otros, los que posteriormente podrán ser comprobados o descartados.

En este contexto, es importante considerar la fuerte influencia que ejerce el capital cultural del individuo (factor interno) en una serie de otras características que se relacionan con la empleabilidad. Dicho capital cultural, al momento de ingresar a la universidad, no sólo ya está consolidado, sino que además influye en otros factores de carácter externo, tales como el establecimiento educacional de origen y el ingreso a universidades de prestigio.

En este escenario los resultados del estudio tanto en sus fases cuantitativas como cualitativas, muestran que las/os profesoras dan cuenta de altos niveles de confianza en sus conocimientos pedagógicos, sin embargo, atribuyen mayor influencia en la permanencia en sus actuales empleos a los conocimientos adquiridos durante el ejercicio de su profesión por sobre los que la universidad les pudo entregar durante su formación. Profundizando, el estudio cualitativo ratificó que las tituladas no dan cuenta de problemas de dominio del contenido disciplinar al momento de enfrentar el primer año de ejercicio de la profesión, no obstante, son enfáticas en señalar su debilidad en la preparación en didáctica de la especialidad y aspectos ligados al ejercicio cotidiano de la profesión, tales como orientación, jefatura, entre otros.

Tomando en consideración los esfuerzos desplegados por el MINEDUC desde fines de la década del 90` con la implementación de los programas fortalecimiento a la profesión docente (Cox, Beca y Cerri 2011; Montecinos et al. 2010), seguido por los proyectos MECE para las Facultades de Educación, la ley 20.129 que estableció la acreditación obligatoria para las carreras de pedagogía y la reciente promulgación de la ley 20.903 que creó el Sistema de Desarrollo Profesional Docente, es preocupante que los profesores sigan atribuyendo mayor importancia en su empleabilidad (entendida como la permanencia en un establecimiento educacional) a las habilidades que adquieren en el ejercicio de su profesión y no a la formación pedagógica recibida. 
Lo anterior supone importantes desafíos para las instituciones responsables de la formación inicial docente, toda vez que los docentes parecen no reconocer su paso por la universidad como un factor relevante en su desempeño profesional, así como tampoco para la obtención de un trabajo. A pesar que de que varias de las entrevistadas, ejercen su primer año de ejercicio docente, en el mismo establecimiento educativo donde realizó su práctica profesional.

Otro de los hallazgos interesantes de este estudio, dice relación con la inserción laboral a partir de un factor no consignado en la literatura, el que se denominó topográfico y que es independiente de las condiciones académicas o de prestigio de la universidad. Las entrevistas en profundidad permitieron dar cuenta que este factor se explica por el hecho de ser ex estudiante de un determinado establecimiento ya sea de enseñanza básica o media, ser oriundo de la zona o ciudad y que se explicó como incidencia de la idiosincrasia, la empatía o familiaridad; en este sentido, se aplican expresiones como, es chilote, es sureño, es penquista, por ejemplo.

Este retorno topográfico y humano, que refuerza las características contextuales y, por tanto externas, no tiene por qué estar contenido en el currículum de FID propiamente tal, pero sí, son necesarias de atender como un aspecto fundamental y determinante por el compromiso de cambio social que tiene todo proyecto educativo universitario.

En este escenario es legítimo preguntarse respecto a la forma cómo las instituciones formadoras de profesores pueden hacerse cargo de las condiciones de empleabilidad de sus titulados y brindar las oportunidades de aprendizaje para que estos mejoren sus competencias y se conviertan en verdaderos agentes del cambio social.

En la actualidad, el foco de la Formación Inicial Docente está en el fortalecimiento de los aspectos pedagógicos (didáctica general y del contenido) y no en el desarrollo explícito de competencias genéricas que apunten a mejorar la empleabilidad de sus egresados. En este sentido, aparentemente, los profesores nóveles que en promedio logran una temprana y exitosa empleabilidad, se debe a las características particulares de su entorno sociocultural, más que a las herramientas dadas por la universidad durante su formación.

Entonces las preguntas que surgen son: ¿Cómo la universidad incorpora las "habilidades individuales" que potencian la empleabilidad? En otras palabras: ¿Cómo brindarles oportunidades de aprendizaje reales a estos estudiantes sin descuidar los aspectos didácticos-disciplinarios del proceso de formación docente?

Desde hace décadas en la FID se ha buscado la fusión de la disciplina con la pedagogía, sin embargo tanto a nivel internacional como nacional, la tendencia es la separación de estos procesos, con departamentos y académicos especialistas para cada área sin considerar las nuevas corrientes en FID que hablan de un diálogo permanente entre los distintos conocimientos que requiere la profesión docente (Shulman 1987). En este contexto, cabe preguntarse ¿Cómo incluir el desarrollo de estas competencias de empleabilidad que parecieran estar determinadas por el contexto y las particularidades de los individuos a un proceso de formación docente estandarizado?

Es preciso mencionar que pese a que los datos son solo representativos para la institución en donde fue aplicado el estudio; la información que de estos se obtuvo permite sin lugar a dudas a otras organizaciones que se ocupan de la formación de profesores(as) fortalecer sus procesos de evaluación y autorregulación.

Finalmente, los autores subrayan que estudios de este tipo pueden orientar a las instituciones responsables de la formación docente a observar sus planes de estudio con 
mayor detención y de esta manera intentar dar respuesta de mejor forma a todas aquellas tensiones que perciben los profesores titulados, en el ejercicio de su profesión.

\section{REFERENCIAS BIBLIOGRÁFICAS}

American Educational Research Association, American Psychological Association \& National Council on Measurement in Education. (2014). The Standards for Educational and Psychological Testing (4th Edition). New York: Harcourt.

Ávalos, B. (2009). La inserción profesional de los docentes. Profesorado Revista de currículum y formación del profesorado, 13 (1), 43-59. Recuperado el 20 de septiembre de 2017 desde http:// www.redalyc.org/pdf/567/56711733004.pdf

Ávalos, B., Carlson, B., y Aylwin, P. (2004). La inserción de profesores neófitos en el sistema educativo: ¿Cuánto sienten que saben y cómo perciben su capacidad docente en relación con las tareas de enseñanza asignadas? Informe proyecto Fondecyt 1020218.

Bransford, J, Brown, A. y Cocking, R. (2000). How People Learn: Brain, Mind, Experience, and School. Washington, D.C. National Academy Press.

Bustos, A., y Coll, C. (2010). Los entornos virtuales como espacios de enseñanza y aprendizaje. Revista Mexicana de Investigación Educativa, 15 (44), 163-184. Recuperado el 17 de octubre de 2017 desde http://www.scielo.org.mx/scielo.php?script=sci_arttext\&pid=S1405-66662010000100009

Cabezas, V., Gallego, F., Santelices, V. y Zarhi, M. (2011). Factores correlacionados con las trayectorias laborales de docentes en Chile, con especial énfasis en sus atributos académicos. Informe proyecto FONIDE $\mathrm{N}^{\circ}$ : FS511082- 2010.

Cornejo, J. (1999). Profesores que se inician en la docencia: algunas reflexiones al respecto desde América Latina. Revista Iberoamericana de Educación, 19 (1), 51-100. Recuperado el 22 de septiembre de 2017 desde https://rieoei.org/RIE/article/view/1055

Corvalán, O. (2010). La profesionalización docente, condición para la carrera docente. Revista Electrónica de Desarrollo de Competencias, 2 (6), 124-151. Recuperado el 8 de octubre de 2017 desde http://redec.utalca.cl/index.php/redec/article/view/54

Cox, C. (2007). Niveles del sistema escolar y formación de profesores: fallas estructurales e implicancias de la inacción. En J.J. Brunner y C. Peña (Ed.), La reforma al sistema escolar: aportes para el debate (pp. 87-101). Santiago: Universidad Diego Portales.

Cox, C., Beca, C., y Cerri, M. (2011). Nueva agenda de políticas docentes en América Latina y El Caribe: Nudos críticos y criterios de acción. Documento de trabajo de la Reunión Regional Ministerial para América Latina y el Caribe del Consejo Económico y Social de las Naciones Unidas (ECOSOC): Buenos Aires. Recuperado el 8 de octubre de 2017 desde http://www. unesco.org/fileadmin/MULTIMEDIA/FIELD/Santiago/pdf/nueva-agenda-Buenos-Aires.pdf

Cox, C., Meckes, L., y Bascopé, M. (2010). La institucionalidad formadora de profesores en Chile en la década del 2000: velocidad del mercado y parsimonia de las políticas. Revista Pensamiento Educativo, 46-47, 205-245. Recuperado el 23 de septiembre de 2017 desde http:// pensamientoeducativo.uc.cl/files/journals/2/articles/468/public/468-1034-1-PB.pdf

Creswell, J (2013). Qualitative inquiry and research design. Choosing Among Five Approaches. Nebraska: SAGE publications.

Darling-Hammond, L. \& Bransford, J. (Eds.) (2005). Preparing teachers for a changing world: What teachers should learn and be able to do. San Francisco: Jossey-Bass.

Geeregat, O. Cifuentes, G. y Villarroel, M. (2016). Factores que inciden en las condiciones de empleabilidad de los egresados en pedagogía. Revista Actualidades Investigativas en Educación, 16(1), 1-19. Recuperado el 23 de septiembre de 2017 desde https://revistas.ucr.ac.cr/index.php/ aie/article/view/21935. DOI:10.15517/aie.v16i1.21935

Haydn, T. \& Barton, R. (2007). Common needs and different agendas: How trainee teachers make 
progress in their ability to use ICT subject. Some lessons from the UK. Journal Computers and Education, 49 (4), 1018-1036. Recuperado el 7 de octubre de 2017 desde http://www.sciencedirect. com/science/article/pii/S0360131506000042 DOI:10.1016/j.compedu.2005.12.006

Hernández, R.; Fernández, C. y Baptista, P. (2010). Metodología de la Investigación (5ta. Ed.). México D.F.: McGrawHill.

Hogan, T. (2004) Pruebas Psicológicas. Una Introducción Práctica (2da Ed.). México D.F.: El Manual Moderno.

Imbernón, F. (2007). La formación y el desarrollo profesional del profesorado. Hacia una nueva cultura profesional (7ma Ed.). Barcelona: Graó.

Latorre, M. (2005). Continuidades y rupturas entre Formación Inicial y Ejercicio Profesional Docente. Revista Iberoamericana de Educación, 36 (2), 1-12. Recuperado el 7 de octubre de 2017 desde https://dialnet.unirioja.es/servlet/articulo?codigo=3130480.

Libaneo, J. (2008). Didáctica y epistemología: para después de la oposición entre la didáctica y las didácticas específicas. En C. D’Avila (Ed.), Profesión docente: nuevos sentidos, nuevas perspectivas. Campinas: PaPIRUS.

Manzi, J. (2010) Programa INICIA: fundamentos y primeros avances. En C. Bellei, D. Contreras y J.P. Valenzuela (Eds.), Ecos de la revolución pingüina (pp.285-308). Santiago: Salesianos Impresiones, S.A.

Meckes, L. y Bascopé, M. (2010). Distribución inequitativa de los nuevos profesores mejor preparados. Ponencia presentada al Primer Congreso Interdisciplinario de Investigación en Educación. Santiago: CIAE-CEPPE. Recuperado el 27 de septiembre de 2017 desde http:// achie.cl/ciie2010/docs/doc/sesiones/249_LMeckes_Distribucion_profesores.pdf

Mendizábal, N. (2006) Los componentes del diseño flexible en la investigación cualitativa. En I. Vasilachis. (Ed). Estrategias de investigación cualitativa. Barcelona: Gedisa S.A.

MIDE-UC (2008). Percepción de la calidad actual de los titulados y graduados de la Educación Superior Chilena. Santiago: Pontificia Universidad Católica de Chile.

Ministerio de Educación, Chile (2005). Informe comisión sobre Formación Inicial Docente. Santiago: Mineduc.

Montecinos, C., Walker, H., Solis, M. C., Núñez, C., Contreras, I., y Rittershaussen, S. (2010). Lineamientos para el diseño del currículum del área de formación de carreras de pedagogía. En S. Martinic y G. Elacqua (Eds.) ¿Fin del ciclo? Cambios en la gobernanza del sistema educativo (pp. 233 - 255). Santiago: UNESCO y Pontifica Universidad Católica de Chile.

Organización para la Cooperación y el Desarrollo Económicos (2004). Revisión de políticas nacionales de educación. París: OCDE.

Pérez, E. y Medrano, L. (2010). Análisis factorial exploratorio: bases conceptuales y metodológicas. Revista Argentina de Ciencias del Comportamiento (RACC), 2 (1), 58-66. Recuperado el 8 de octubre de 2017 desde http://www.redalyc.org/html/3334/333427068006/

Rojas, F., Chávez, K., y Suazo, P. (2011). Transición desde el mundo universitario al mundo profesional: redes, necesidades y competencias genéricas en estudiantes de la universidad de La Frontera. Temuco: Universidad de La Frontera.

Ruffinelli, A. (2013) La calidad de la Formación Inicial Docente en Chile: La Perspectiva de los Profesores Principiantes. Revista Calidad en la educación, 39, 117-154. Recuperado el 6 de octubre de 2017 de http://www.scielo.cl/scielo.php?pid=S0718-45652013000200005 \&script=sci_arttext. DOI: $10.4067 /$ S0718-45652013000200005

Ruffinelli, A., y Guerrero, A. (2009). Círculo de segmentación del sistema educativo chileno: Destino laboral de egresados de Pedagogía en Educación Básica. Revista Calidad en la Educación, 31, 19-44. Recuperado el 6 de octubre de 2017 desde https://www.nuevo.cned.cl/sites/default/files/ revista/cse_articulo832.pdf

Shulman, L. (1987). Knowledge and teaching: Foundations of the new reform. Harvardeducational review, 57 (1), 1-23. Recuperadoel3 de agosto de 2017 desde http://www.hepgjournals.org/doi/abs/10.17763/ 
haer.57.1.j463w79r56455411?code=hepg-site. DOI:10.17763/haer.57.1.j463w79r56455411

Strauss, A., Corbin, J., y Zimmerman, E. (2002). Bases de la investigación cualitativa: técnicas y procedimientos para desarrollar la teoría fundamentada. Medellín: Universidad de Antioquia.

Vonk, J. (1996). A Knowledge base for mentors of beginning teachers: results of a dutch experience. in Mc. Bride (Ed.), Teacher education policy: some issues arisign from research and practice (pp. 112-134). London: Farmer Press.

Walsh, K. (2005). IcT's about Learning: School leadership and the effective integration of information and communications technology. Melton Mowbray: King Edward VII School 